\title{
Clinical Profile of Critical Pertussis in Children at a Pediatric Intensive Care Unit in Northern India
}

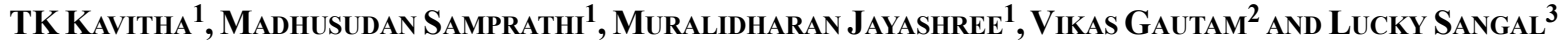 \\ From ${ }^{1}$ Pediatric Emergency and Intensive Care Units, ${ }^{2}$ Medical Microbiology, Post Graduate Institute of Medical Education and \\ Research, Chandigarh, India; and ${ }^{3}$ NPO-VPD laboratories, WHO Country Office for India.
}

\author{
Correspondence to: Dr Muralidharan \\ Jayashree, Professor, Pediatric \\ Emergency and Intensive Care Units, \\ Post Graduate Institute of Medical \\ Education and Research, Chandigarh \\ 160012 , India. \\ Received:January 12, 2019; \\ Initial review: June 08, 2019; \\ Accepted: November 08, 2019
}

\begin{abstract}
Objective: To delineate the clinical profile, complications, intensive care needs, and predictors of mortality in children with critical pertussis. Methods: Retrospective analysis of case records of children in the pediatric intensive care unit of a tertiary-care hospital, with a diagnosis of critical pertussis over 3 years. Diagnostic criteria included CDC case definition and confirmation by polymerase chain reaction (PCR), when available. Survivors and nonsurvivors were compared to identify predictors of mortality. Results: 36 records were analysed, most cases were infants (31, 86.1\%). 10 (27.7\%) were (below 6 weeks of age). In the rest, $16(61.5 \%)$ were partially immunized or unimmunized against pertussis. Rapid breathing (88.9\%), paroxysmal cough (86.1\%) and apnea $(41.7 \%)$ were common presenting complaints. Hypoxemia (97.2\%), hyperleukocytosis $(61.1 \%)$ and encephalopathy $(52.8 \%)$ were common complications. Intensive care needs were mechanical ventilation in 11 $(30.6 \%)$, vasoactive support in $7(19.4 \%)$ and exchange transfusion in $3(8.3 \%)$. Female gender, apnea, hyperleukocytosis, encephalopathy, need for vasoactive support, and mechanical ventilation predicted mortality. Conclusion: Pertussis demands attention due to its varied presentation, increased complications and higher mortality.
\end{abstract}

Key words: Apnea, DPT vaccine, Immunization, Outcome.
$\mathrm{P}$ ertussis has shown an alarming increase in global incidence recently [1]. Disease burden is high despite vaccination coverage of almost $85 \%$ among children [2]. Precise data from lowand middle-income countries (LMICs) are unavailable due to variable case definitions, limited awareness, inadequate infrastructure and weak surveillance systems. The exact cause of resurgence is unclear. Multiple factors like antigenic shifts in bacteria, waning vaccine immunity and reduced duration of protection by acellular pertussis vaccine have been implicated [1]. Studies suggest that source of infection may be identifiable only in about $27-43 \%$ of infant pertussis, and the most common source is usually mothers or siblings [3-5]. Critical pertussis is defined as pertussis requiring admission to an intensive care unit (ICU) or resulting in death [6]. It can lead to life-threatening complications like pulmonary arterial hypertension (PAH), respiratory failure and shock. Mortality rate ranges from 4.8-55\% [713]. Data on critical pertussis is scarce, with very few reports from LMICs like India [13,14]. In this case series we describe the clinical profile, complications, intensive care needs and predictors of mortality in children with critical pertussis.

\section{METHODS}

This was a retrospective study in the Pediatric Intensive Care Unit of a tertiary-level teaching and referral hospital in Northern India. Cases were identified from the electronic database of the unit over a period of 3 years (2016-18), and those fulfilling the clinical and critical pertussis criteria as per WHO case definition were included. Clinical pertussis was defined as 'any patient with cough lasting $\geq 2$ weeks with at least one of the following symptoms - paroxysmal cough, inspiratory whoop or post-tussive vomiting [2]. Critical pertussis was defined as pertussis requiring admission to an ICU or resulting in death. A total of 36 children with critical pertussis were retrieved for final analysis. No children were excluded. Demographic details (age, immunization status), presenting complaints, intensive care needs, complications, laboratory parameters and treatment modalities were recorded on a pre-designed performa. Hyperleukocytosis was defined as total leukocyte count (TLC) $>50,000 / \mu \mathrm{L}$ [15]. Real time polymerase chain reaction (PCR) targeting IS481 and Ptxs1 was done in 28 children. Serology using IgM ELISA antipertussis toxin was also done in 21 children. All children received 
azithromycin $10 \mathrm{mg} / \mathrm{kg} /$ day for 5 days and other supportive measures, which included respiratory and vasoactive support, and exchange transfusion depending on clinical need.

Statistical analysis: Chi-square and Fisher's exact tests were used to compare proportions while Student $t$ test and Mann Whitney test were used for means. Survivors and non-survivors were compared by univariate analysis to identify predictors of mortality. SPSS version 21 was used for statistical analysis.

\section{RESULTS}

Thirty-six cases (58.5\% boys) of critical pertussis were enrolled. Median (IQR) age was $3.5(1.5,7)$ months. Most children were infants $(n=31,86.1 \%)$, with $10(27.7 \%)$ being below six weeks and too young to be immunized against pertussis. The rest $16(61.5 \%)$ were partially immunized or unimmunized against pertussis. Contact history with respiratory illness was evident in only two children. The major presenting symptoms were rapid breathing in $32(88.9 \%)$, typical paroxysmal cough in 31 $(86.1 \%)$ and apnea in $15(41.7 \%)$ children. Most patients $(35,97.2 \%)$ had hypoxemia $\left(\mathrm{SPO}_{2}<94 \%\right.$ in room air $)$ at admission.

Hyperleukocytosis and thrombocytosis were noted in $22(61.1 \%)$ and $26(72.2 \%)$ patients, respectively. Median (IQR) total leukocyte count (TLC) (per $\mu \mathrm{L}$ ) was 64,000 (23050, 100037). Out of 28 children tested for pertussis RT-PCR, $19(52.8 \%)$ were positive. Of the 8 children in whom PCR was not done, 1 was positive, 4 showed intermediate positivity and 3 were negative by ELISA. When tested for co- infections with other viruses, two children were positive for RSV, and all were negative for $\mathrm{H} 1 \mathrm{~N} 1$.

Hypoxemia (35, 97.2\%) was the commonest complication, followed by hyperleukocytosis, encephalo-pathy $(19,52.8 \%)$, seizures $(17,47.2 \%)$, and acute kidney injury $(6,16.6 \%)$. Out of 11 children who under-went neuroimaging, multiple CNS infarcts were seen in 3 children and 8 were normal. Pulmonary arterial hyper-tension (PAH) was seen in 5 of 15 children who under-went echocardiography $(33.3 \%)$. Intensive care needs were mechanical ventilation in 11 (30.6\%), vasoactive support in $7(19.4 \%)$ and exchange transfusion (ET) in $3(8.3 \%)$. The most common indication for intubation was apneic spells in 9 children, out of which 4 were emergent during spells, and remaining 5 were elective for recurrent spells and hypoxemic events. Two children were intubated for encephalopathy. In majority $(n=6)$, the intubation was done within 24 hours. The problems faced during ventilation were recurrent apnea $(n=9)$, paroxysms of cough $(n=9)$, air leaks $(n=2)$, and ventilator associated pneumonia $(n=1)$. Of the 7 ventilated children who underwent echocardiography, 4 had pulmonary arterial hypertension (PAH). Persistent hypoxemia and failure of conventional ventilation was seen in four children who required high frequency ventilation. Healthcare associated infections were seen in four children; ventilator-associated pneumonia in one child and blood stream infections in three children.

An increasing trend of hospital incidence ( 2 cases in 2016, 7 in 2017 and 27 in 2018), and mortality (no mortality in 2016, 1 in 2017 and 7 in 2018) of critical pertussis was observed over the 3 year study period. Eight patients $(22.2 \%)$ with median (IQR) age of $3.5(1.1,5.5)$ months died; all were unimmunised including three who being $<6$ weeks old had yet to begin primary immunisation. All non survivors except one required mechanical ventilation. Major causes of death were hypoxemia and refractory shock (4), massive brain infarcts (2), secondary infection (1), and acute kidney injury and hyperkalemia ( $n=1$ each). Female gender $(P=0.04)$, apnea $(P=0.01)$, hyperleukocytosis $(P=0.01)$, encephalopathy (Glasgow coma score $<14)(P=0.04)$, need for vasoactive support $(P<0.001)$ and mechanical ventilation $(P<0.001)$ were significantly associated with mortality on univariate analysis. PAH and need for ET did not determine mortality (Table I).

\section{DISCUSSION}

Our study has shown an increased hospital incidence of critical pertussis in young infants before completion of their primary vaccination. Major complications noted were hypoxemia, hyperleukocytosis, encephalopathy and seizures. PAH was present in few children. Intensive care needs were ventilation, vasoactive support and exchange transfusion. Female gender, apnea, hyperleukocytosis, encephalopathy, need for vasoactive support and mechanical ventilation were predictors of mortality.

The study: however, suffers from the inherent drawbacks of a retrospective analysis. Diagnostic tests like PCR could not be done in all. Details of maternal immunization status were unavailable and leukocyte count threshold for initiating exchange transfusion was not clearly defined.

Incidence of pertussis is increasing globally with periodic outbreaks being reported from different parts of the world including India [16-19]. Resurgence of a vaccine preventable disease like pertussis causing increasing hospitalization, costs and mortality is a 


\section{What This Study Adds?}

- There was a high prevalence of seizures, encephalopathy, hyperleukocytosis and pulmonary artery hypertension in this cohort of critical pertussis compared to earlier studies.

Table I Predictors of Mortality in Children with Critical Pertussis $(N=36)$

\begin{tabular}{llllr}
\hline Predictors of mortality & Survivors $(n=28)$ & Non-survivors $(n=8)$ & Odds ratio $(C I)$ & $P$ value \\
\hline Male gender & $19(67.8)$ & $2(25)$ & $0.15(0.02-0.94)$ & 0.043 \\
Apnea & $14(50)$ & $8(100)$ & $2(1.38-2.89)$ & 0.01 \\
Hyperleukocytosis & $14(50)$ & $8(100)$ & $2(1.38-2.89)$ & 0.01 \\
*Peak leucocyte count, per $\mu \mathrm{L}$ & $44900(17225-91357)$ & $100025(68200-144512)$ & - & 0.002 \\
Encephalopathy $(\mathrm{GCS}<14)$ & $12(42.8)$ & $7(87.5)$ & $2.04(1.23-3.37)$ & 0.04 \\
Vasoactive support needed & $1(3.5)$ & $6(75)$ & $21(2.94-149.95)$ & $<0.001$ \\
Mechanical ventilation & $4(14.2)$ & $7(87.5)$ & $6.12(2.38-15.74)$ & $<0.001$ \\
Exchange transfusion & $1(3.5)$ & $2(25)$ & $7(0.72-67.63)$ & 0.12 \\
\hline
\end{tabular}

All values in $n$ (\%) except *median (IQR); GCS: Glasgow coma scale.

worrisome trend, and has led to calls for relook of immunization schedules [16,20]. However, our patients were mostly young infants similar to previously published reports $[8,13]$. Over half of the patients were unimmunized against pertussis. Critical pertussis occurring before primary immunization highlights the importance of maternal immunization against pertussis. Pertussis masquerading as any other acute respiratory infection often runs the risk of under-diagnosis and under-reporting. None of our patients were suspected to have pertussis nor received macrolide antibiotics before referral. Delayed diagnosis can make 'benign' pertussis 'critical' due to evolving complications. The prevalence of seizures, encephalopathy, hyperleukocytosis in this cohort was significantly higher compared to earlier studies on critical pertussis which have reported seizures in $9-16 \%$, encephalopathy in nearly $20 \%$ and hyperleukocytosis in 21-35\% of children [8-13]. Onethird of the screened patients had PAH. Increased pulmonary vascular resistance is postulated to be secondary to obstruction of pulmonary vasculature by lymphocytes accumulation resulting from hyperleukocytosis [21]. Leucocytosis, especially in young infants, was shown to be associated with PAH, encephalopathy, greater risk of PICU admission and mortality but a causal link is yet to be proven $[10,15]$. Therefore screening of all patients of critical pertussis for PAH is essential. Children with critical pertussis often require mechanical ventilation and inotropic support for hypoxemia, apnoea, shock, PAH and encephalopathy [13]. Exchange transfusion for hyperleukocytosis especially if done before organ failure or immediately at the onset of shock was found to be associated with improved hemodynamics, hypoxemia and mortality in few case series and reports; the clear cut indications and mortality benefits are yet to be conclusively proven on a larger scale [14,22,23]. Mortality in critical pertussis varies between 4.8-55\% [7-13]. Recognized predictors of mortality include younger age, comorbidities, need for ventilation, vasoactive use, PAH and a rapid course, similar to those identified in this study [7-13].

We have highlighted the important complications; intensive care needs and our limited experience with exchange transfusion in patients with hyperleukocytosis. To conclude, resurgence of pertussis demands attention due to its varied presentation, increased complications and higher mortality. The importance of clinical recognition and empirical treatment in such a setting cannot be overemphasised. Prospective studies on critical pertussis, its complications and the utility of various therapies are the need of the hour.

Contributors: TKK, MS: collected and analysed the data and prepared the initial draft of the manuscript; MJ designed the study, finalized the manuscript; VG and LS provided the laboratory support and gave critical inputs for the manuscript. All authors approved the final version.

Funding: None; Competing interests: None stated.

\section{REFERENCES}

1. Jackson DW, Rohani P. Perplexities of pertussis: Recent global epidemiological trends and their potential causes. Epidemiol Infect. 2014;142:672-84. 
2. World Health Organization. Disease surveillance and Burden. Available from: www.who.int/immunization/moni toring-surveillance/burdens//en. Accessed on November 27, 2019.

3. Bisgard KM, Pascual FB, Ehresmann KR, Miller CA, Cianfrini C, Jennings CE, et al. Infant pertussis: Who was the source? Pediatr Infect Dis J. 2004;23:985-9.

4. Kowalzik F, Barbosa AP, Fernandes VR, Carvalho PR, Avila-AgueroML, Goh DY, et al. Prospective multinational study of pertussis infection in hospitalized infants and their household contacts. Pediatr Infect Dis J. 2007;26:238-42.

5. Skoff TH, Kenyon C, Cocoros N, Liko J, Miller L, Kudish $\mathrm{K}$, et al. Sources of infant pertussis infection in the United States. Pediatrics. 2015;136:635-41.

6. Burr JS, Jenkins TL, Harrison R, et al. The Collaborative Pediatric Clinical Care Research Network (CPCCRN) Critical Pertussis Study: Collaborative research in pediatric critical care medicine. Pediatr Crit Care Med. 2011;12:38792.

7. Borgi A. Predictors of mortality in mechanically ventilated critical pertussis in a low income country: new resurgence in 2013. Mediterr J Hematol Infect Dis. 2014;6:e2014059.

8. Kazantzi MS, Prezerakou A, Kalamitsou SN, Ilia S, Kalabalikis PK, Papadatos J, et al. Characteristics of Bordetella pertussis infection among infants and children admitted to paediatric intensive care units in Greece: A multicentre, 11-year study. J Paediatr Child Health. 2017;53:257-62.

9. Palvo F, Fabro AT, Cervi MC, Aragon DC, Ramalho FS, Carlotti APCP. Severe pertussis infection. Medicine (Baltimore). 2017;96:e8823.

10. Chong C-Y, Yung C-F, Tan NW-H, Acharyya S, Thoon KC. Risk factors of ICU or high dependency requirements amongst hospitalized pediatric pertussis cases: A 10 year retrospective series, Singapore. Vaccine. 2017;35:6422-8.

11. Straney L, Schibler A, Ganeshalingham A, Alexander J, Festa M, Slater A, et al. Burden and outcomes of severe pertussis infection in critically ill infants. Pediatr Crit Care Med. 2016;17:735-42.
12. Macdonald-Laurs E, Ganeshalingham A, Lillie J, McSharry B, Segedin ER, Best E, et al. Increasing incidence of life-threatening pertussis. Pediatr Infect Dis J. 2017;36:282-9.

13. Berger JT, Carcillo JA, Shanley TP, Wessel DL, Clark A, Holubkov R, et al. Critical Pertussis Illness in Children. Pediatr Crit Care Med. 2013;14:356-65.

14. Rauf A, Sachdev A, Gupta D. Exchange transfusion as a treatment strategy in severe pertussis with hyperleukocytosis in an infant - A case report. Critical Care Pediatrics. 2018;1:1-3.

15. Mikelova LK, Halperin SA, Scheifele D, Smith B, FordJones E, Vaudry W, et al. Predictors of death in infants hospitalized with pertussis: a case-control study of 16 pertussis deaths in Canada. J Pediatr. 2003;143:576-81.

16. van der Maas NAT, Mooi FR, de Greeff SC, Berbers GA, Spaendonck MA, de Melker HE. Pertussis in the Netherlands, is the current vaccination strategy sufficient to reduce disease burden in young infants? Vaccine. 2013;31:4541-7.

17. Etkind P. Pertussis outbreaks in the United States. Am Fam Physician. 1991;44:1622-3.

18. Dahiya S, Kapil A, Kabra SK, Mathur P, Sood S, Lodha $\mathrm{R}$, et al. Pertussis in India. J Med Microbiol. 2009;58: 688-9.

19. Takum T, Gara D, Tagyung H, Murhekar M V. An outbreak of pertussis in Sarli Circle of Kurung-kumey district, Arunachal Pradesh, India. Indian Pediatr. 2009;46:1017-20.

20. Vashishtha VM. Adolescent immunization schedule: Need for a relook. Indian Pediatr. 2019;56:101-04.

21. Peters MJ. Mechanisms of pulmonary hypertension in Bordetella pertussis: Authors' reply. Arch Dis Child. 2003;88:92-b-93.

22. Donoso AF, Cruces PI, Camacho JF, Le??n JA, Kong JA. Exchange transfusion to reverse severe pertussis-induced cardiogenic shock. Pediatr Infect Dis J. 2006;25:846-8.

23. Nieves D, Bradley JS, Gargas J, Mason WH, Lehman D, Lehman SM, et al. Exchange blood transfusion in the management of severe pertussis in young infants. Pediatr Infect Dis J. 2013;32:698-9. 